\title{
Estimation of Time-Varying Pilot Model Parameters
}

\author{
Peter M.T. Zaal* and Barbara T. Sweet ${ }^{\dagger}$ \\ NASA Ames Research Center, Moffett Field, CA
}

\begin{abstract}
Human control behavior is rarely completely stationary over time due to fatigue or loss of attention. In addition, there are many control tasks for which human operators need to adapt their control strategy to vehicle dynamics that vary in time. In previous studies on the identification of time-varying pilot control behavior wavelets were used to estimate the time-varying frequency response functions. However, the estimation of time-varying pilot model parameters was not considered. Estimating these parameters can be a valuable tool for the quantification of different aspects of human time-varying manual control. This paper presents two methods for the estimation of time-varying pilot model parameters, a two-step method using wavelets and a windowed maximum likelihood estimation method. The methods are evaluated using simulations of a closed-loop control task with time-varying pilot equalization and vehicle dynamics. Simulations are performed with and without remnant. Both methods give accurate results when no pilot remnant is present. The wavelet transform is very sensitive to measurement noise, resulting in inaccurate parameter estimates when considerable pilot remnant is present. Maximum likelihood estimation is less sensitive to pilot remnant, but cannot detect fast changes in pilot control behavior.
\end{abstract}

\section{Nomenclature}

$\begin{array}{llll}A_{t} & \text { sinusoid amplitude, deg } & T_{c} & \text { vehicle dynamics time con } \\ e & \text { tracking error signal, deg } & T_{l} & \text { pilot lead time constant, } \\ f & \text { probability density function, - } & T_{m} & \text { measurement time, s } \\ f_{t} & \text { target forcing function, deg } & T_{n} & \text { pilot remnant time constant } \\ H & \text { Heaviside step function } & t & \text { time, s } \\ H_{c} & \text { vehicle dynamics } & u & \text { pilot control signal, deg } \\ H_{o l} & \text { open-loop dynamics } & y & \text { controlled system output } \\ H_{p} & \text { pilot visual response } & W & \text { wavelet transform, }- \\ J & \text { cost function value, - } & w & \text { Gaussian white noise sign } \\ j & \text { imaginary unit } & x & \text { time sequence, }- \\ K_{c} & \text { vehicle dynamics gain, - } & & \\ K_{n} & \text { pilot remnant gain, - } & \alpha y m b o l s & \\ K_{v} & \text { pilot visual gain, - } & \\ k & \text { sinusoid index } & \epsilon & \text { line search parameter, - } \\ M_{\Theta \Theta} & \text { Fisher information matrix } & \phi_{t} & \text { sinusoid phase shift, rad } \\ N & \text { number of points } & \varphi_{m} & \text { phase margin, deg } \\ n & \text { pilot remnant signal, deg } & \eta & \text { nondimensional time, }- \\ n_{t} & \text { forcing function frequency integer factor } & \lambda & \text { wavelet scale, } \mathrm{s} \\ P_{n} & \text { remnant/control signal power ratio,- } & \Theta & \text { parameter vector } \\ s & \text { Laplace variable } & & \end{array}$

${ }^{*}$ Ph.D. candidate, Control and Simulation Division, Faculty of Aerospace Engineering, Delft University of Technology, P.O. Box 5058, 2600GB Delft, The Netherlands; p.m.t.zaal@tudelft.nl. Student member AIAA.

${ }^{\dagger}$ Aerospace Engineer, Human Systems Integration Division, NASA Ames Research Center, Moffett Field, CA, 94035; barbara.t.sweet@nasa.gov. Member AIAA. 


$\begin{array}{llll}\sigma & \text { standard deviation } & \omega_{t} & \text { sinusoid frequency, rad s } \\ \tau_{v} & \text { pilot visual delay, s } & \omega_{0} & \text { Morlet wavelet parameter, - } \\ \omega & \text { frequency, rad s } & \\ \omega_{c} & \text { crossover frequency, rad s } & & \text { wavelet function, - } \\ \omega_{n m} & \text { pilot neuromuscular frequency, } \mathrm{rad} \mathrm{s}^{-1} & \psi_{0} & \text { mother wavelet function, - } \\ & & \zeta_{n m} & \text { pilot neuromuscular damping, - }\end{array}$

\section{Introduction}

Techniques for the identification and prediction of single- and multi-loop time-invariant human control behavior are well established. ${ }^{1-4}$ In order for these techniques to provide accurate identification results, specific requirements are posed on the design of experiments in which human control behavior is estimated. These requirements ensure that the human controller behaves like a stationary control element in the control loop. However, in real-life control tasks, human control behavior is rarely completely stationary over time due to fatigue or loss of attention. In addition, there are many control tasks for which human operators need to adapt their control strategy to vehicle dynamics that vary in time. Examples are the manual control during mode transitions in tilt-rotor aircraft, and the manual control of rotorcraft with variable speed rotors or aircraft with reconfigurable flight control systems. ${ }^{5}$

In the past decades, significant research has been devoted to the modeling of time-variant pilot control behavior. ${ }^{6}$ However, most of this research was focused on the development of complex mathematical models to describe time-varying behavior and not on the estimation of time-varying frequency responses or timevarying model parameters. An accurate estimate of these parameters allows for a quantification of the change in pilot control behavior over time and gives valuable insight into how a pilot adapts to time-varying controlled dynamics or environmental variables. Wavelet transforms have been used in many different applications such as, geophysical applications - to analyze localized variations of power within a time series, ${ }^{7,8}$ but have only recently been introduced into the field of systems and control.

In recent years, work on the identification of time-varying pilot control behavior focused on the use of wavelet transforms to identify time-varying frequency response functions and derived parameters such as crossover frequencies and phase margins. ${ }^{9,10}$ However, several difficulties arise when using wavelets. First, there are many types of wavelets to consider, each with their advantages and disadvantages in terms of energy localization, rise time, and smoothness in the time and frequency domains. In addition, each wavelet has one or more parameters that define its properties in the time-frequency plane. The type of wavelet and its parameters need to be selected by the researcher, making this technique not straightforward to use. ${ }^{11}$ Second, there are insufficient techniques to fully asses the accuracy (bias and variance) of the estimated pilot frequency responses.

In previous studies on the identification of time-varying pilot control behavior using wavelets, the estimation of time-varying pilot model parameters was not considered. ${ }^{9,10}$ When attempting to estimate time-varying model parameters using wavelets a second step is required, fitting the pilot model frequency response to the wavelet transform frequency response. This is similar to the two-step methods used for the estimation of time-invariant pilot model parameters. ${ }^{3}$ A technique exists to estimate the model parameters from time-domain data in a single step using maximum likelihood estimation (MLE), ${ }^{4}$ significantly reducing bias and variance compared to the two-step methods. However, this technique has not been used for the estimation of time-varying parameters in the field of manual control.

This paper presents the results of a study on the estimation of time-varying pilot model parameters to quantify time-varying human control behavior. Two methods are considered. The first is a two-step method using wavelets for the identification of the time-varying pilot frequency responses in the first step. The second method is the use of MLE to estimate the time-varying parameters directly from time-domain data in a single step. A simulation with time-varying pilot and system dynamics was set up to evaluate both methods. The paper is structured as follows. First, the pilot control behavior identification problem will be discussed in Sec. II. The parameter estimation methods using wavelets and MLE will be discussed in Sec. III and Sec. IV, respectively. Next, the simulation setup will be given in Sec. V, followed by the identification results in Sec. VI. Finally, a discussion and conclusions will be given in Sections VII and VIII, respectively. 


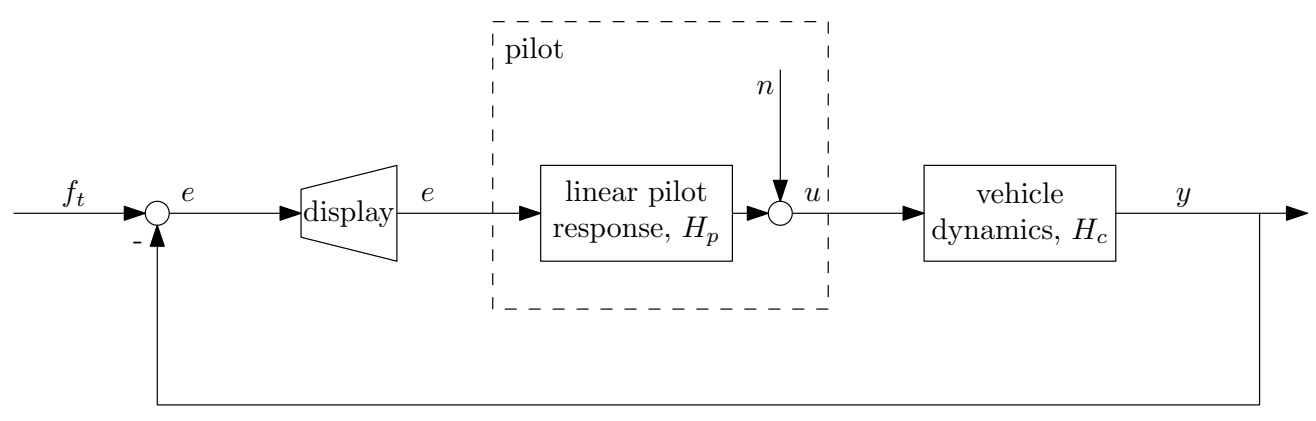

Figure 1: Closed-loop compensatory control task.

\section{Identification of Pilot Control Behavior}

The human operator is a non-linear biological system. However, in a continuous control task - when trained properly and given constant environmental conditions - the operator's manual control behavior can be described by a quasi-linear time-invariant model and a remnant signal that accounts for non-linear behavior. ${ }^{1}$ This cybernetic approach to characterize human control behavior has been a powerful research tool to investigate the effects of different perceptual cues on pilot control behavior, ${ }^{12}$ assess aircraft handling qualities ${ }^{13}$ and evaluate different control system designs. ${ }^{14}$

A single-loop compensatory control task is presented in Figure 1. In this control task, a pilot is actively controlling the vehicle dynamics $H_{c}$, while following a target signal $f_{t}$. The error signal $e$ that is the difference between the target forcing function $f_{t}$ and the vehicle dynamics output $y$ is presented on a compensatory display. This task setup allows for the identification of a single frequency response function $H_{p}$ that defines pilot control behavior. The output of this linear response function combined with a remnant signal $n$ that accounts for non-linear behavior, defines the pilot's control output $u$.

To excite the combined pilot-vehicle system for the identification of pilot control behavior, it is common to use a forcing function $f_{t}$ that is defined as a multi-sine signal. These signals have power at distinct input frequencies that cover the frequency range of human control. To reduce the difficulty of the control task and to lower the probability of crossover regression, the number of input frequencies is usually limited to 12 and the power at higher input frequencies is reduced. ${ }^{1}$

Current methods, used to characterize pilot control behavior, are based on the concept that the human controller is a linear time-invariant control element in the control loop. However, human control behavior is rarely time invariant, due to factors like fatigue or loss of attention. This means the linear pilot frequency response function $H_{p}$ is a function of time. Furthermore, there are many control tasks where human operators need to adapt their control strategy to vehicle dynamics $H_{c}$ that change in time, for example, manual control during mode transitions in tilt-rotor aircraft or manual control during a failure of aircraft flight control system components. Finally, the visual properties of cockpit instruments or displays might change in time, for example, due to a change in environmental lighting conditions.

When using linear time-invariant system identification techniques, small variations of pilot control behavior in time are captured in the remnant signal. However, when these variations are more significant and are the subject of study, identification techniques that are capable of capturing time-variant system dynamics need to be used. In previous research on the identification of time-varying pilot control behavior, the wavelet transform was used to identify time-varying pilot frequency response functions and derived parameters, such as crossover frequencies and phase margins. ${ }^{9,10}$ However, the estimation of time-varying pilot model parameters was not considered.

Two different techniques to estimate the time-varying pilot model parameters are evaluated in this paper. An overview of these techniques is given in Figure 2. The estimation of the time-varying model parameters using wavelets requires two steps. In the fist step, a time-varying frequency response is identified using the wavelet transform. In the second step, the model parameters are estimated at each time step by fitting a pilot model to the frequency response. Using MLE to estimate the time-varying pilot model parameters requires only one step. The parameters are estimated directly using the time-domain data. A sliding time window will be used to estimate the parameters at each time step. These two parameter estimation techniques are discussed further in the following sections. 


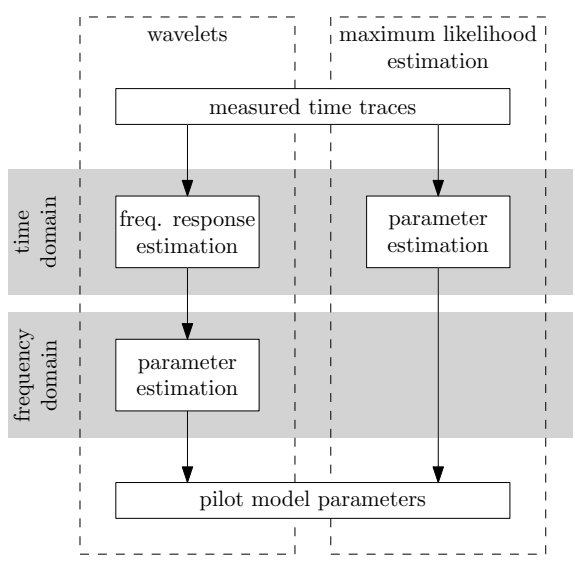

Figure 2: Identification methods for the estimation of time-varying pilot model parameters from measured time traces.

\section{Wavelet Transform Parameter Estimation}

This section describes the estimation of time-varying model parameters using the wavelet transform. The wavelet transform is used to identify a time-varying frequency response in the first step, followed by a pilot model fit to estimate the model parameters in the second step. Both steps will be discussed in this section.

\section{A. Step 1: The Wavelet Transform}

The wavelet transform can be used to analyze a time series that contains time-variant power at different frequencies. Numerous good references exist that give a complete overview of the continuous and discrete wavelet transforms, the different types of wavelet functions, and a large number of examples in different applications. ${ }^{7,8,11}$ This section provides a brief overview of the continuous wavelet transform and the Morlet wavelet as used in this study. Notations and equations are adopted from Ref. 8.

The continuous wavelet transform of a discrete time signal $x_{n}$, with $n=0,1, \ldots, N-1$ and $N$ the number of points in the time series, is defined as the convolution of $x_{n}$ with a wavelet function $\psi$ that is a scaled and translated version of a mother wavelet $\psi_{0}$ :

$$
W(\lambda)=\sum_{m=0}^{N-1} x_{m} \psi^{*}\left[\frac{(m-n) \delta t}{\lambda}\right]
$$

with $\delta t$ the sampling interval and $\lambda$ the wavelet scale. $*$ indicates the complex conjugate. By varying the wavelet scale $\lambda$ and translating along the localized time index $n$, the magnitude information can be constructed as a function of scale and time. If complex wavelets are used, magnitude and phase information can be constructed. When the scale $\lambda$ increases, the wavelet becomes more spread out in time and takes only low-frequency features of the time sequence into account, and vice versa.

The convolution in Eq. (1) is performed $N$ times for each scale, which is a computational intensive procedure. The continuous wavelet transform can be approximated by performing the calculations in Fourier space using the Fourier transform, making the procedure considerably faster. Using the convolution theorem, the continuous wavelet transform can be approximated by:

$$
W(\lambda) \approx \sum_{k=0}^{N-1} X_{k} \Psi^{*}\left(\lambda \omega_{k}\right) e^{i \omega_{k} n \delta t}
$$

where $X$ and $\Psi$ are the Fourier transforms of $x$ and $\psi$, respectively. $k$ is the frequency index and $\omega_{k}$ the angular frequency defined by:

$$
\omega_{k}=\frac{2 \pi k}{N \delta_{t}}(k \leq N / 2) \text { and } \omega_{k}=-\frac{2 \pi k}{N \delta_{t}}(k>N / 2)
$$


(a) time domain

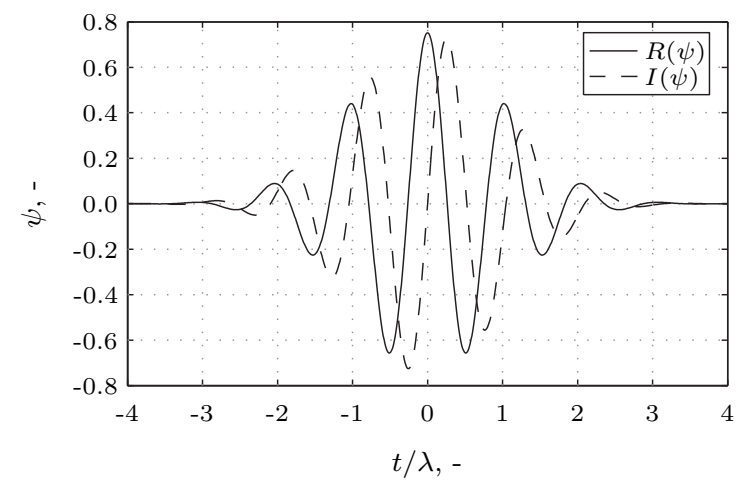

(b) frequency domain

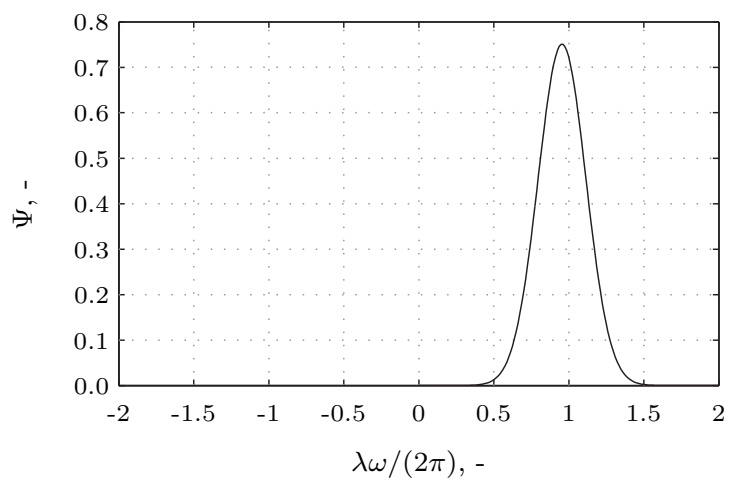

Figure 3: The Morlet wavelet function $\left(\omega_{0}=6\right.$ and $\left.\lambda=10 \delta t\right)$.

Using Eq. (2), the continuous wavelet transform for a given scale can be calculated simultaneously for the entire time sequence with $N$ points. The wavelet function $\Psi$ in Eq. (2) needs to be normalized to have unit energy at each scale $\lambda$ to ensure that each wavelet transform is weighted only by the amplitude of $X$ and not by the wavelet function itself. This allows for the wavelet transforms at each scale to be directly comparable. The normalization is defined by:

$$
\Psi\left(\lambda \omega_{k}\right)=\left(\frac{2 \pi s}{\delta t}\right)^{1 / 2} \Psi_{0}\left(\lambda \omega_{k}\right)
$$

There are many types of functions that can be used as mother wavelets. Commonly used functions for the continuous wavelet transform are the Morlet, Paul, and Mexican hat. A valid wavelet function must have zero mean and unit energy $\left(\int_{-\infty}^{\infty}\left|\Psi_{0}(\omega)\right|^{2} d \omega=1\right)$. Furthermore, the function must have compact support or sufficiently fast decay to obtain localization in both the time and frequency domains.

One of the most widely used continuous wavelets is the complex Morlet wavelet, which consists of a wave signal modified by a Gaussian envelope. The Morlet wavelet is used in the remainder of this report, as it has the property that it minimizes the time-frequency localization. ${ }^{10}$ Furthermore, because of the complex nature of this wavelet, it is able to detect both time-dependent amplitude and phase information at different frequencies, which is a requirement for system identification applications such as the identification of pilot control behavior. The Morlet wavelet is defined by:

$$
\psi_{0}(\eta)=\pi^{-1 / 4} e^{i \omega_{0} \eta} e^{-\eta^{2} / 2}
$$

with $\omega_{0}=12$ the nondimensional frequency, and $\eta=t / \lambda$ a nondimensional time parameter. The real and imaginary parts of this wavelet function are depicted in Figure 3a. The Fourier transform of the Morlet wavelet function is given by Eq. (6), in which $H(\omega)$ is the Heaviside step function. The frequency response function of the Morlet wavelet function is depicted in Figure 3b.

$$
\Psi_{0}(s \omega)=\pi^{-1 / 4} H(\omega) e^{-\left(\lambda \omega-\omega_{0}\right)^{2} / 2}
$$

Using the wavelet transform defined in Eq. (2), it is necessary to choose a set of scales $\lambda$. For a given type of wavelet function, a relationship between scales and frequencies can be derived analytically by substituting a cosine wave of a known frequency into Eq. (2) and deriving the scale at which the wavelet power spectrum reaches its maximum. Using this approach for the Morlet wavelet function, scales can be related to frequencies using the following expression:

$$
\lambda=\frac{\omega_{0}+\sqrt{2+\omega_{0}^{2}}}{2 \omega}
$$

with $\omega$ the frequency in radians per second.

As the power of the forcing function is limited to certain input frequencies, the linear pilot response function can only be identified at these frequencies. This means the continuous wavelet transform only needs 
to be calculated at the input frequencies of the forcing function. This can be accomplished by calculating the scales corresponding to the forcing function input frequencies using Eq. (7). Using these scales, the time-varying pilot frequency response function can be approximated by:

$$
\hat{H}_{p}(j \omega, t)=\frac{W_{u}(\lambda)}{W_{e}(\lambda)}
$$

where $W_{u}$ and $W_{e}$ are the wavelet transforms of $u$ and $e$, respectively, defined by Eq. (2). For the Morlet wavelet function, the scales are related to the input frequencies of the forcing function by Eq. (7). The same approach can be taken to estimate the time-varying frequency response function of the controlled dynamics $H_{c}(j \omega, t)$ or the time-varying open-loop response $H_{o l}(j \omega, t)$.

\section{B. Step 2: Pilot Model Fit}

The parameters of a pilot model are estimated by fitting the model to the estimated time-varying frequency response function from Eq. (8) by minimizing a weighted least-squares criterion using a constrained gradientbased optimization. The cost function used in the current study is defined by:

$$
J(\Theta)=\frac{1}{N_{t}} \sum_{k=1}^{N_{t}} \frac{\left|\hat{H}_{p}(j \omega)-\tilde{H}_{p}(j \omega, \Theta)\right|^{2}}{\left|\hat{H}_{p}(j \omega)\right|^{2}}
$$

where $N_{t}$ is the number of input frequencies where the estimated pilot frequency response function is defined and $\Theta$ is the vector of parameters to be estimated. This criterion quantifies the difference between the estimated pilot frequency response function $\hat{H}_{p}$ and the frequency response function of the pilot model $\tilde{H}_{p}$. By repeating this procedure at every time step where the pilot frequency response function is defined, the change of the pilot model parameters in time can be determined. To reduce the computational effort needed to calculate the time-varying parameters, the number of calculations can be reduced by resampling the timevarying frequency response to a lower sampling rate in time. In the current study, the initial parameters for the optimization problem are the (to be estimated) simulated pilot model parameters.

\section{Maximum Likelihood Parameter Estimation}

Maximum likelihood estimation has been used in previous research to estimate the parameters of timeinvariant pilot models. ${ }^{15}$ An extensive description of MLE for the estimation of pilot model parameters is given in Reference 4. This section gives a brief description of the MLE procedure adopted in this paper.

MLE attempts to find an estimate $\hat{\Theta}$ of the parameter vector $\Theta$ that maximizes the likelihood function. The likelihood function $L(\Theta)$ is defined as the joint conditional probability density function of the prediction error for $m$ measurements of the pilot control signal $u(k)$ :

$$
L(\Theta)=f(\epsilon(1), \epsilon(2), \ldots, \epsilon(k), \ldots, \epsilon(m) \mid \Theta)
$$

The prediction error, indicated as $\epsilon(k)$ in Eq. (10), is defined as the difference between the measured pilot control signal $u(k)$ and the modeled pilot control signal $\hat{u}(k)$ at discrete instants. When the remnant is assumed to be an additive zero-mean Gaussian white noise signal, the conditional probability density function for one measurement of $\epsilon(k)$ is given by:

$$
f(\epsilon(k) \mid \Theta)=\frac{1}{\sqrt{2 \pi \sigma_{n}^{2}}} e^{-\frac{\epsilon^{2}(k)}{2 \sigma_{n}^{2}}}
$$

The set of parameters that maximizes the likelihood function is the maximum likelihood estimate of the parameter vector $\Theta$. For the MLE method it is common practice to minimize the negative natural logarithm of the likelihood function instead of maximizing $L(\Theta)$, as this results in a more straightforward optimization problem. When a global minimum of the negative log-likelihood is attained, the resulting parameter vector is the maximum likelihood estimate, indicated with $\hat{\Theta}_{M L}$. For a single-output pilot model, combining Eq. (10) and Eq. (11) results into the following expression for the maximum likelihood parameter estimate: 


$$
\hat{\Theta}_{M L}=\underset{\Theta}{\arg \min }-\ln L(\Theta)=\underset{\Theta}{\arg \min }\left[\frac{m}{2} \ln \sigma_{n}^{2}+\frac{1}{2 \sigma_{n}^{2}} \sum_{k=1}^{m} \epsilon^{2}(k)\right]
$$

The minimum of this function is found using an unconstrained gradient-based Gauss-Newton optimization. The iterative parameter update equation for the Gauss-Newton optimization is given by:

$$
\hat{\Theta}(i+1)=\hat{\Theta}(i)-\alpha(i) M_{\Theta \Theta}^{-1}(\hat{\Theta}(i)) \frac{\partial L(\hat{\Theta}(i))}{\partial \Theta}
$$

where $\alpha$ is the line-search parameter. This parameter, which typically varies between 0 and 1 , is determined at each iteration before the actual parameter update to ensure the most rapid minimization of the likelihood function. The gradients of the likelihood function with respect to all parameters, $\partial L / \partial \Theta$, can be evaluated using the Jacobians of the pilot model state-space matrices with respect to the parameter vector $\Theta$. The Fisher information matrix, indicated with the symbol $M_{\Theta \Theta}$ in Eq. (13), is given by:

$$
M_{\Theta \Theta}=\frac{1}{\sigma_{n}^{2}} \sum_{k=1}^{m}\left(\frac{\partial \epsilon(k)}{\partial \Theta}\right)^{2}
$$

The MLE optimization requires the pilot models to be written in output-error state-space form. These state-space representations are easily obtained by converting the transfer function models using the controller canonical form. For the conversion to a state-space representation, the pilot model time delays are approximated using fifth order Padé approximations. Due to these Padé approximations, the matrices of the state-space model contain coefficients that are highly nonlinear functions of the pilot model parameters.

To estimate time-varying pilot model parameters in the current study, the MLE optimization is performed at every time step $t_{i}$ using a sliding time window of length $\Delta t$. This is visualized in Figure 4. Choosing a $\Delta t$ that is too small will decrease the accuracy of estimated parameters related to low-frequency dynamics. A $\Delta t$ that is too large will reduce the method's ability to detect small variations in pilot model parameters. In the current study, the length of the time window is chosen to be $20 \mathrm{~s}$.

In Reference 4, to increase the likelihood of finding a global optimum solution, the initial parameter set for the Gauss-Newton optimization is determined by a genetic optimization procedure. This procedure requires a considerable amount of computational power, especially when performed at every time step. To reduce the computational effort in the current study, the initial parameter set was constructed from the simulated pilot model parameters at every time step.

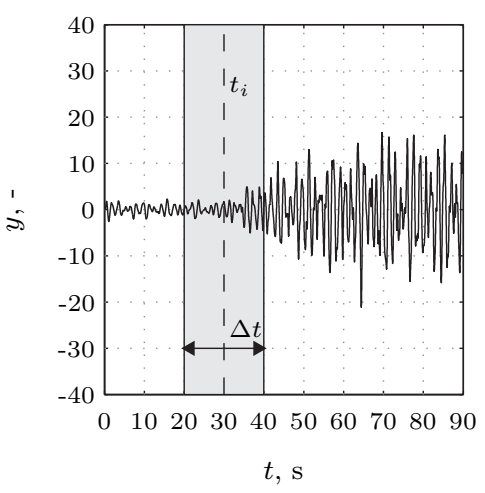

Figure 4: MLE time window.

\section{Simulation Setup}

A simulation was set up in Matlab ${ }^{\circledR}$ and Simulink ${ }^{\circledR}$ to allow for an evaluation of the estimation of time-varying pilot model parameters using wavelets and MLE. This section describes the control task, timevarying dynamics, and forcing function and remnant signals used in the simulation. Values for time-varying parameters used in the simulation are not directly taken from previous experiment data. However, the parameters were adjusted manually to approximate pilot performance results of previous studies.

\section{A. Control Task}

Figure 5 depicts the simulated closed-loop target-following control task. The task of the pilot is to minimize the error $e$ - that is, the difference between the target forcing function $f_{t}$ and the system output $y$ - visible on a compensatory display. The time-varying vehicle and pilot dynamics are given by $H_{c}(s, t)$ and $H_{p}(s, t)$, respectively. The pilot control signal $u$ serves as the input to the controlled vehicle dynamics and is the summation of the linear pilot response function output and a remnant signal $n$ that accounts for non-linear behavior.

The time-varying vehicle dynamics are given by: 


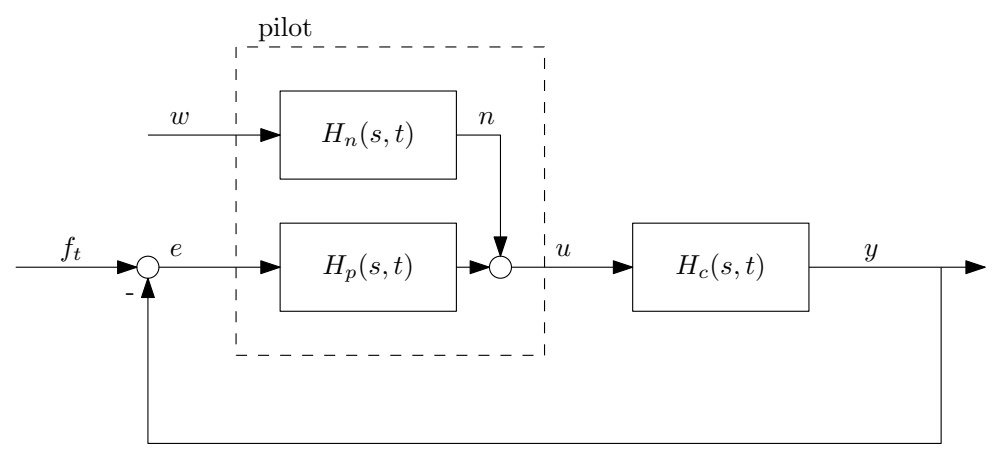

Figure 5: Simulated closed-loop control task with time-varying dynamics.

$$
H_{c}(s, t)=\frac{K_{c}(t)}{T_{c}(t) s^{2}+s}
$$

where $K_{c}(t)$ is a time-varying gain and $T_{c}(t)$ is a time-varying time constant. These dynamics are a single integrator below the break frequency of $1 / T_{c}(t) \mathrm{rad} / \mathrm{s}$ and a double integrator above this frequency.

The time-varying pilot dynamics are determined by:

$$
H_{p}(s, t)=K_{v}(t)\left(1+T_{l}(t) s\right) e^{-s \tau_{v}} \frac{\omega_{n m}^{2}}{\omega_{n m}^{2}+2 \zeta_{n m} \omega_{n m} s+s^{2}}
$$

The equalization dynamics of the pilot consist of a time-varying gain $K_{v}(t)$ and a time-varying lead time constant $T_{l}(t)$. The time delay $\tau_{v}$, and the neuromuscular parameters $\zeta n m$ and $\omega_{n m}$, represent the pilot's limitations and are constant in the current simulation.

The pilot remnant signal $n$ was simulated by passing a zero-mean Gaussian white noise signal through a time-varying low-pass filter:

$$
H_{n}(s, t)=\frac{K_{n}(t)}{T_{n} s+1}
$$

with a time-varying gain $K_{n}(t)$ and a fixed time constant $T_{n}$.

\section{B. Time-Varying Dynamics}

A single simulation run with a length of $90 \mathrm{~s}$ is divided into three parts. In the first part, until $t_{1}=30 \mathrm{~s}$, the controlled vehicle and pilot dynamics are constant. Next, until $t_{2}=70 \mathrm{~s}$, the dynamics transition from one state to the other by linearly changing the time-varying parameters $K_{c}(t), T_{c}(t), K_{v}(t), T_{l}(t)$, and $K_{n}(t)$. After the transition phase the dynamics remain constant again for the remainder of the simulation run.

The values for the time-varying parameters for the length of a run are given in Figure 6 . The fixed values for the pilot model time delay, and neuromuscular damping and frequency, are set to $\tau_{v}=0.20 \mathrm{~s}, \zeta_{n m}=0.20$, and $\omega_{n m}=10 \mathrm{rad} / \mathrm{s}$, respectively. Note that the values for the lead time constants of the vehicle and pilot model dynamics are equal throughout the run. This means that a perfect pilot compensation is assumed for the double integrator vehicle dynamics at higher frequencies; that is, the open-loop dynamics $\left(H_{o l}=H_{p} H_{c}\right)$ have single integrator characteristics for the entire frequency range.

Frequency responses of the time-varying vehicle, pilot and open-loop dynamics for every $5.0 \mathrm{~s}$ in the transition phase are given in Figure 7. As can be observed from Figure 7a, the vehicle dynamics change from mostly integrator dynamics to mostly double integrator dynamics. As a result of this transition, the pilot needs to generate more lead, as can be observed in Figure 7b. Note that despite the fact that the parameters change linearly, the magnitude and phase of the vehicle and pilot responses do not change linearly. Both magnitude and phase change more rapidly at the beginning of the transition phase.

\section{Target Forcing Function}

The target forcing function is a sum of sines constructed using the following equation: 


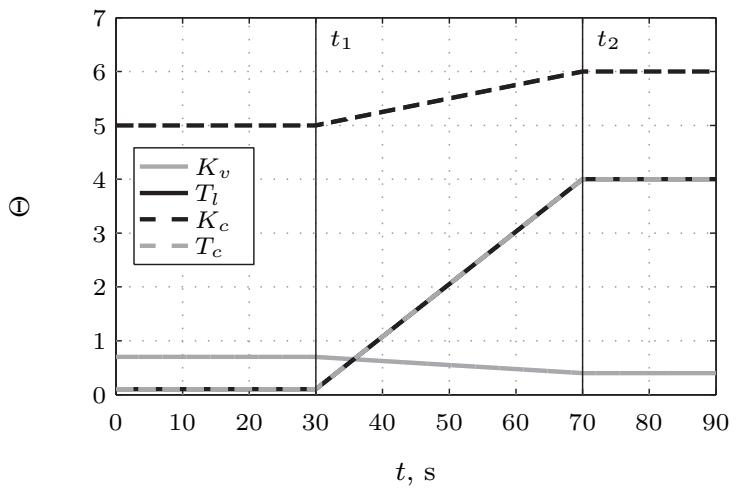

Figure 6: Values of the time-varying simulation parameters.
Table 1: Forcing function properties.

\begin{tabular}{rrclc}
\hline \hline$k,-$ & $n_{t},-$ & $\omega_{t}, \mathrm{rad} \mathrm{s}^{-1}$ & $A_{t}, \mathrm{deg}$ & $\phi_{t}, \mathrm{rad}$ \\
\hline 1 & 5 & 0.384 & 0.629 & -2.571 \\
2 & 13 & 0.997 & 0.629 & -1.059 \\
3 & 27 & 2.071 & 0.629 & 1.736 \\
4 & 41 & 3.145 & 0.629 & 2.060 \\
5 & 53 & 4.065 & 0.629 & -2.790 \\
6 & 73 & 5.599 & 0.063 & -1.221 \\
7 & 103 & 7.900 & 0.063 & 2.020 \\
8 & 139 & 10.661 & 0.063 & 0.127 \\
9 & 194 & 14.880 & 0.063 & 1.483 \\
10 & 229 & 17.564 & 0.063 & -0.537 \\
\hline \hline
\end{tabular}

(a) vehicle magnitude

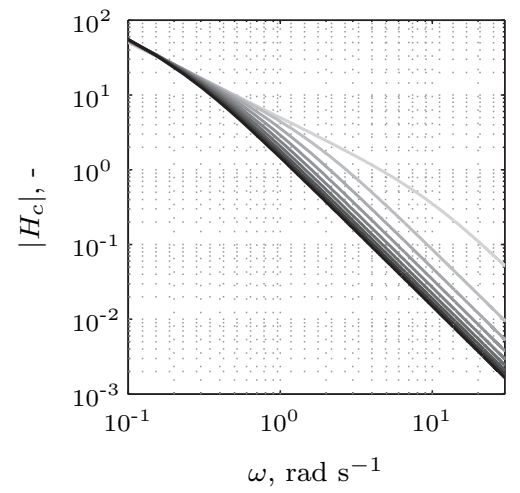

(d) vehicle phase

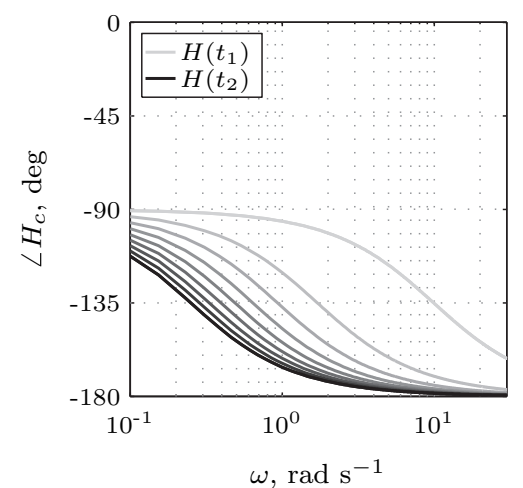

(b) pilot magnitude

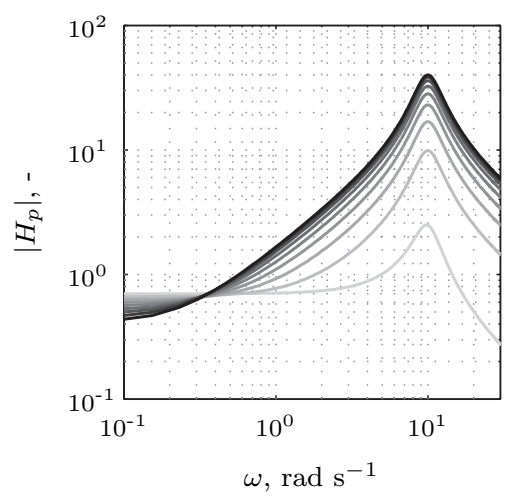

(e) pilot phase

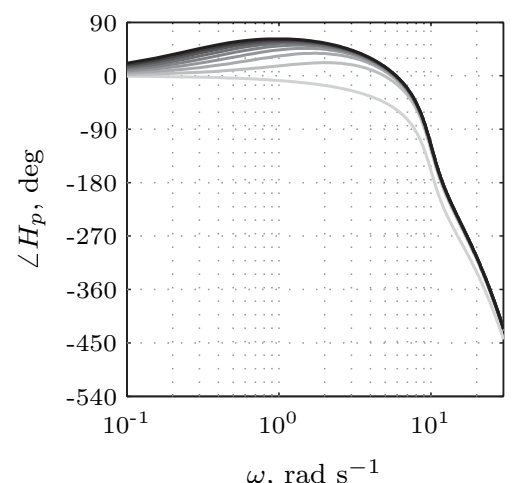

(c) open-loop magnitude

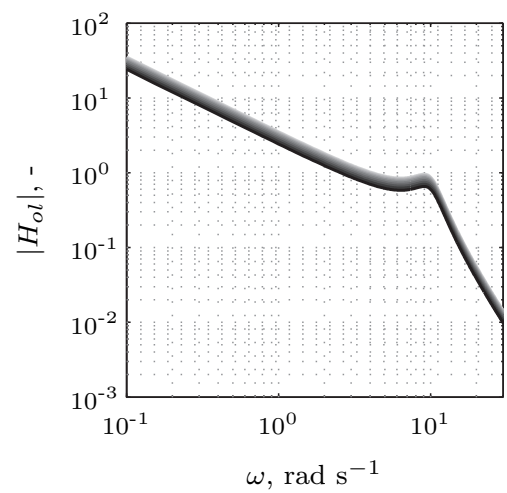

(f) open-loop phase

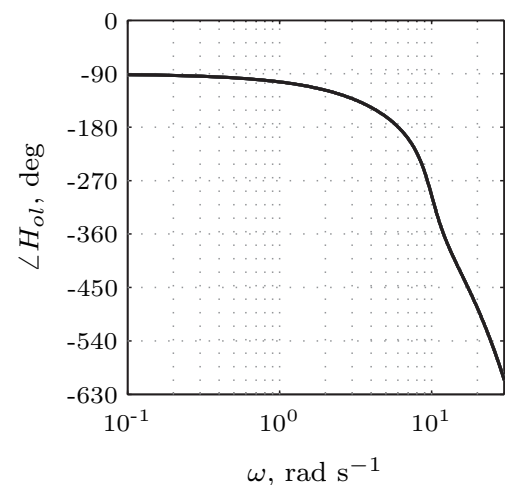

Figure 7: Frequency responses of the time-varying vehicle, pilot, and open-loop dynamics from $t_{1}=30 \mathrm{~s}$ until $t_{2}=70 \mathrm{~s}$ for every $5.0 \mathrm{~s}$. 
(a) error signal

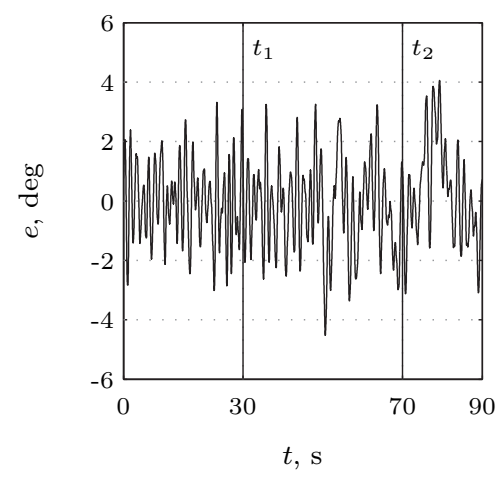

(b) control signal

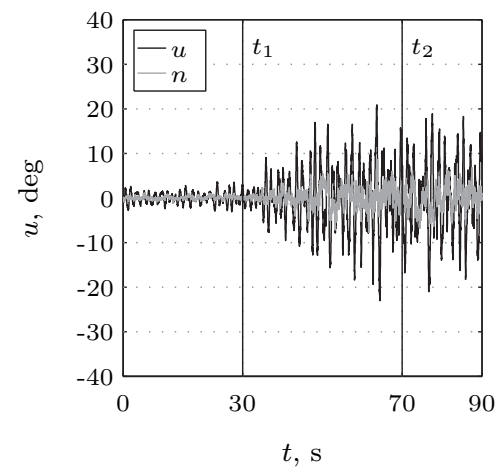

(c) state signal

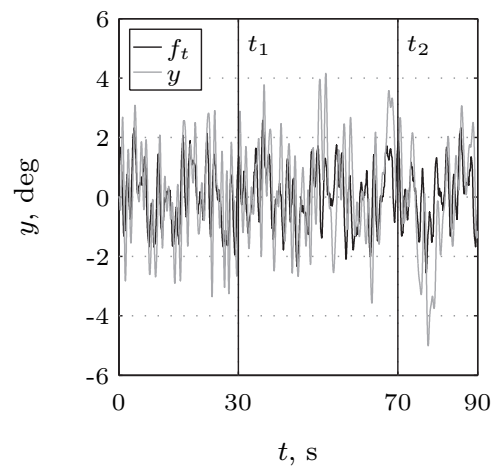

Figure 8: Time traces of the simulation signals.

$$
f_{t}(t)=\sum_{k=1}^{N_{t}} A_{t}(k) \sin \left[\omega_{t}(k) t+\phi_{t}(k)\right]
$$

with $N_{t}=10$ the number of sine waves, and $\omega_{t}, A_{t}$ and $\phi_{t}$ the frequency, amplitude and phase shift of the $k^{\text {th }}$ sine wave, respectively. The measurement time used to construct the forcing function was set to $T_{m}=81.92 \mathrm{~s}$. With a simulation sampling frequency of $100 \mathrm{~Hz}$, this measurement time contains the highest power-of-two data points. The sinusoid frequencies $\omega_{t}(k)$ were all integer multiples of the measurement-time base frequency, $\omega_{m}=2 \pi / T_{m}=0.0767 \mathrm{rad} / \mathrm{s}$, and were covering the frequency range of human control (0.1-20 $\mathrm{rad} / \mathrm{s})$. The amplitudes for the lowest five frequencies were set equal and the amplitudes at the remaining frequencies were set to one tenth of the low frequency amplitudes. The forcing-function phase distribution was randomly generated. The final forcing-function signal was scaled to have a variance of $1.0 \mathrm{deg}$. Table 1 provides a summary of all the forcing-function properties.

\section{Pilot Remnant}

The value of the constant remnant filter time constant $T_{n}$ was set to $0.2 \mathrm{~s}$. The time-varying gain $K_{n}(t)$ was calculated to induce a certain pre-set power ratio between remnant and pilot control signal $\left(P_{n}=\sigma_{n}^{2} / \sigma_{u}^{2}\right)$ for the entire run. The gain can be calculated using the following expression for the variance of a signal $x$ :

$$
\sigma_{x}^{2}=\frac{1}{\pi} \int_{0}^{+\infty} S_{x x}(\omega) d \omega=\frac{1}{\pi N} \int_{0}^{+\infty} X^{*}(\omega) X(\omega) d \omega
$$

Using this expression to calculate the variances of $n$ and $u$, and substituting the frequency responses of these signals as defined by Equations 20 and 21, the value of $K_{n}(t)$ can be calculated as a function of the time-varying pilot and controlled dynamics.

$$
\begin{gathered}
N(j \omega)=H_{n}(j \omega) W(j \omega) \\
U(j \omega)=\frac{H_{p}(j \omega)}{1+H_{p}(j \omega) H_{c}(j \omega)} F_{T}(j \omega)+\frac{H_{n}(j \omega)}{1+H_{p}(j \omega) H_{c}(j \omega)} W(j \omega)
\end{gathered}
$$

Time traces of the different signals in the control loop for a single simulation run are given in Figure 8. In this simulation run, the remnant was scaled to be $10 \%$ of the total pilot control signal $\left(P_{n}=0.1\right)$, using the above equations.

\section{Results}

This section provides the results calculated using the simulation discussed in Sec. V. Simulations with and without pilot remnant signals were performed to investigate the influence of pilot remnant on the estimates of pilot control behavior and the pilot model parameters. 
(a) WVL pilot magnitude

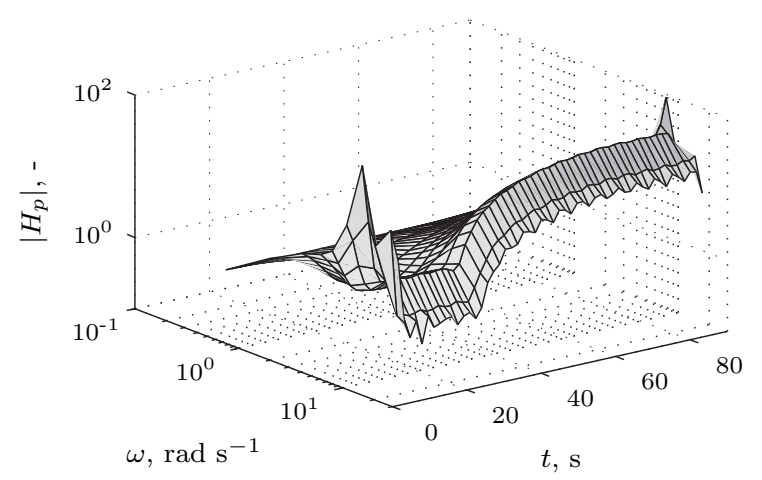

(c) WVL pilot phase

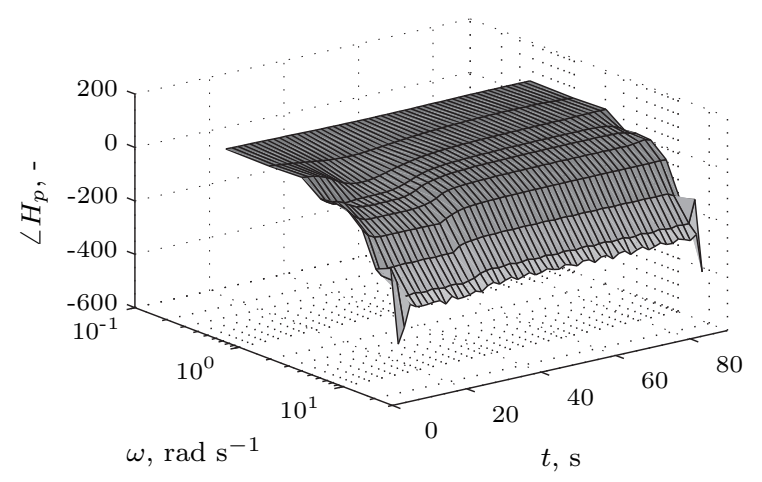

(b) MLE pilot magnitude

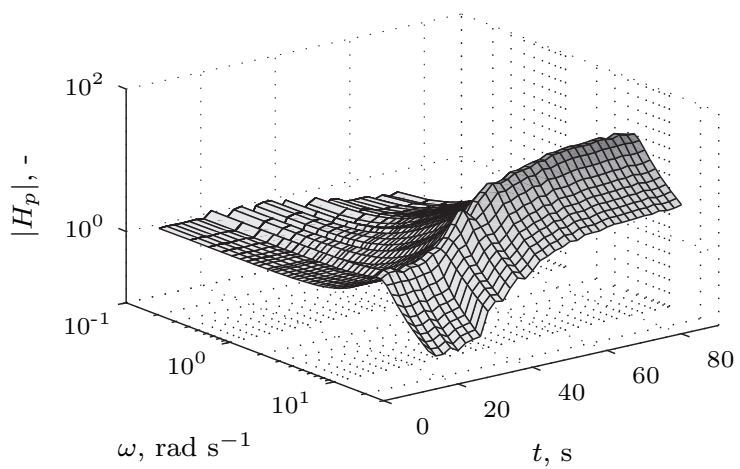

(d) MLE pilot phase

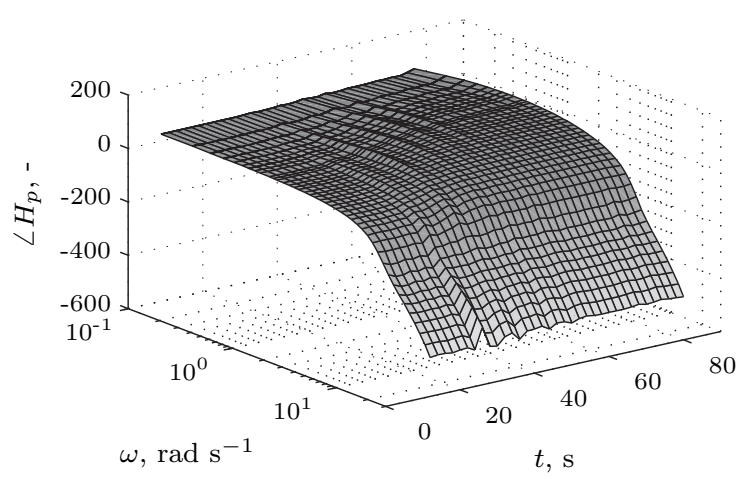

Figure 9: Time-varying pilot identification results from the wavelet and MLE methods $\left(P_{n}=0\right)$.

\section{A. Pilot Control Behavior}

Figure 9 depicts the time-varying pilot frequency responses identified using the continuous wavelet transform and MLE. For the wavelet calculations, only the last $81.92 \mathrm{~s}$ of a simulation run were used; that is, the largest power of two data points. The MLE procedure was performed every two seconds, starting at $t=12.0 \mathrm{~s}$ until $t=80.0 \mathrm{~s}$. Note that a time trace of $20.00 \mathrm{~s}$ was used for the estimation of the pilot frequency response at each time step. The time-domain data used for the identification was taken from a simulation where no pilot remnant was simulated $\left(P_{n}=0\right)$. The figure clearly indicates the time-varying nature of the pilot frequency response.

For the frequency response identified using wavelets, some edge effects can be observed at $t=0.0 \mathrm{~s}$ and $t=81.92 \mathrm{~s}$ in both the magnitude and phase plots, Figures $9 \mathrm{a}$ and $9 \mathrm{c}$, respectively. In addition, some oscillatory behavior of the wavelet transform can be observed in time at higher frequencies. Note that the time-varying wavelet frequency response is only defined at the input frequencies of the forcing function.

The magnitude and phase of the pilot frequency responses identified using MLE are given in Figures 9b and $9 \mathrm{~d}$, respectively. The frequency response appears to be smoother, as knowledge of the pilot model is incorporated into the estimate. By comparing the wavelet and MLE magnitude responses around $t=30.0$ $\mathrm{s}$, it can be observed that the rapid change in the pilot frequency response at the start of the parameter transition is not captured very accurately using MLE. The maximum likelihood estimate shows a more gradual change due to the sliding time window. The sliding time window effectively produces an average frequency response over the length of the time window. At the end of the transition phase at $t=70.0 \mathrm{~s}$, where the rate of change in dynamics is more gradual, the MLE procedure is capable of accurately estimating the change in dynamics.

To better assess the accuracy of the wavelet and MLE estimates and to allow for a better comparison between the two methods, the estimation results are given at three time instances in Figure 10. The time instances coincide with the start, middle, and end points of the parameter transition phase. The pilot frequency response data is equivalent to the data presented in Figure 9. It can be observed that the pilot 
(a) pilot magnitude at $t=30 \mathrm{~s}$

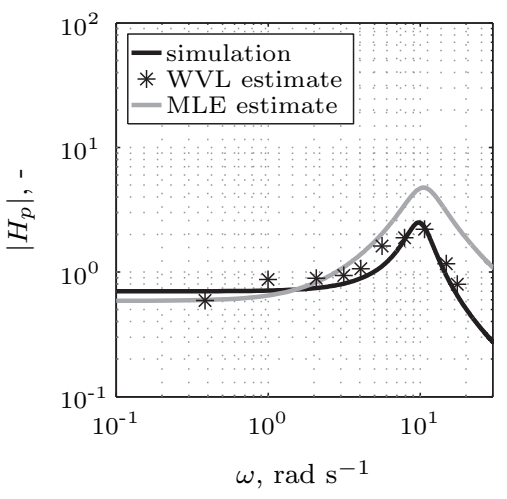

(d) pilot phase at $t=30 \mathrm{~s}$

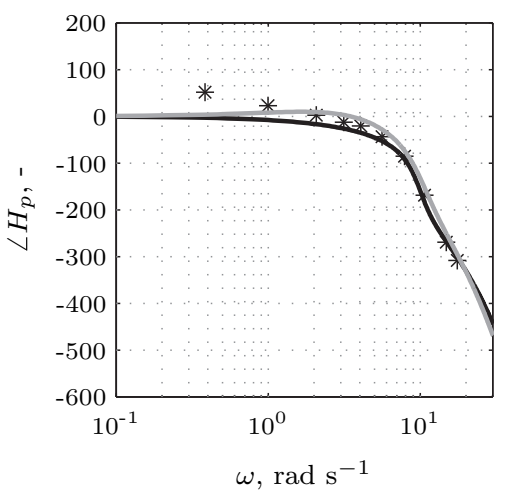

(b) pilot magnitude at $t=50 \mathrm{~s}$

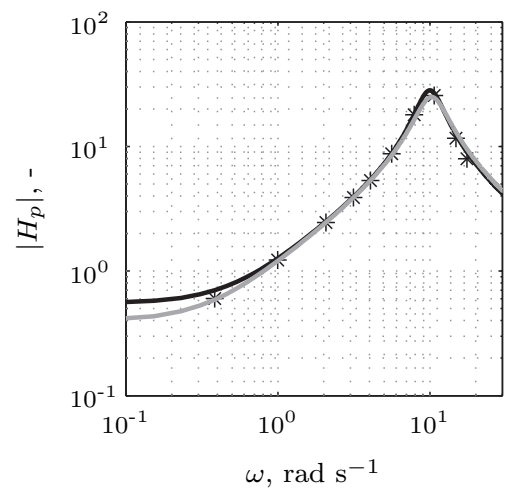

(e) pilot phase at $t=50 \mathrm{~s}$

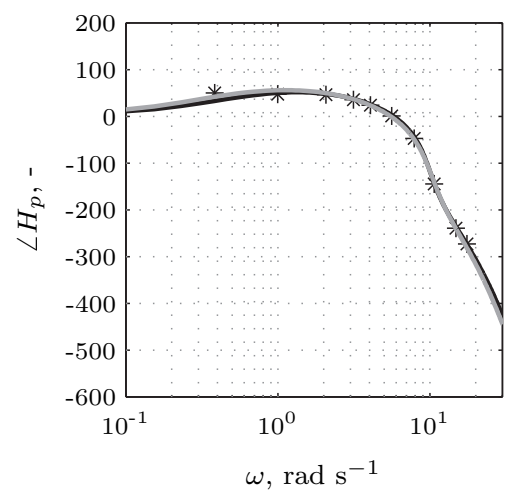

(c) pilot magnitude at $t=70 \mathrm{~s}$

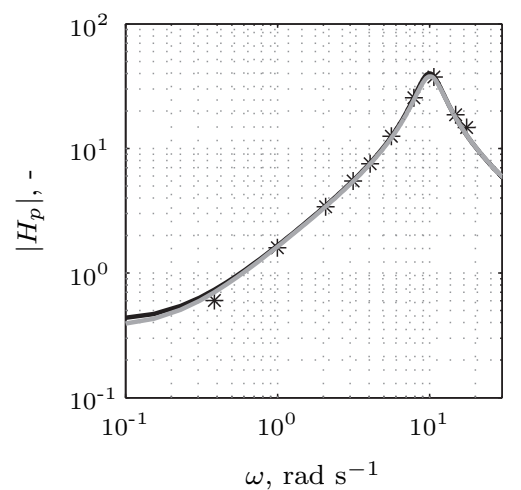

(f) pilot phase at $t=70 \mathrm{~s}$

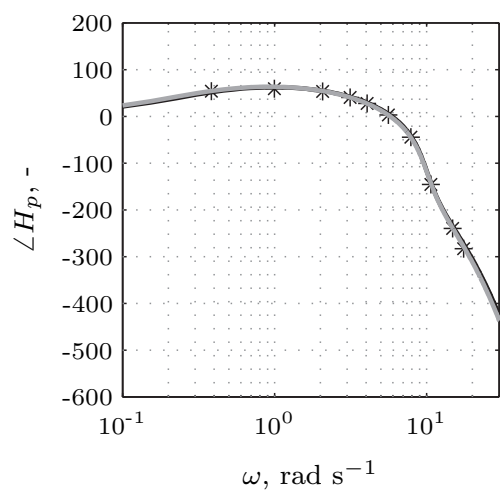

Figure 10: Pilot identification results at different time instances $\left(P_{n}=0\right)$.

frequency response estimates from the wavelet transform - only defined at the input frequencies of the forcing function - follow the simulated pilot response very closely at the given time instances. The estimate from the MLE procedure at $t=30.0 \mathrm{~s}$ does not follow the simulated response very accurately. This is the same effect as observed in Figure 9. The MLE frequency response estimates at the other two time instances accurately follow the simulated responses. Note that the data is from a simulation without simulated remnant.

Figure 11 depicts the estimated frequency responses for a simulation with added pilot remnant. The variance of the remnant signal $n$ was scaled to $5 \%$ of the pilot control signal variance $\left(P_{n}=0.05\right)$. The remnant percentage was kept constant over the length of the simulation run using the procedure described in Sec. V.D. When comparing the wavelet and MLE ferquency responses, it can be observed that the wavelet estimates are most heavily affected by the addition of a remnant signal. A remnant variance percentage of $5 \%$ of the pilot control signal is relatively low. When averaging data runs from an experiment with human subjects, the average remnant variance percentage is above $10 \%$.

\section{B. Crossover Frequencies and Phase Margins}

Figure 12 provides the estimated time-varying crossover frequencies and phase margins from the open-loop responses calculated with the pilot frequency response estimates from Figure 9. The values calculated from the simulated pilot and controlled dynamics are given for reference. For the calculation of the crossover frequency - where the magnitude of the open loop is 1.0 - a single interpolation is required. For the determination of the phase margin - the phase difference with 180 deg at the crossover frequency - another interpolation is required. The errors between the estimated and the simulated crossover frequencies and phase margins are a result of inaccuracies from the frequency response estimates and the interpolation procedures. Note that the MLE frequency response estimates have a much higher resolution in the frequency domain compared to the wavelet estimates, reducing the interpolation errors.

The crossover frequency and phase margin estimates resulting from the wavelet frequency responses 
(a) WVL pilot magnitude

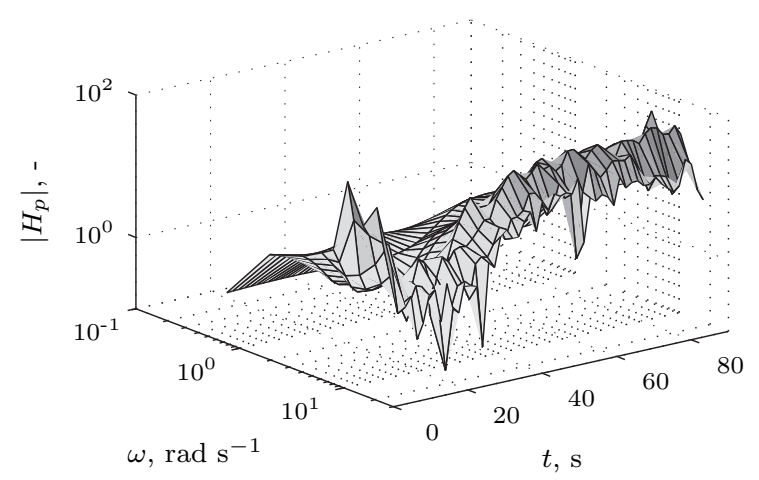

(c) WVL pilot phase

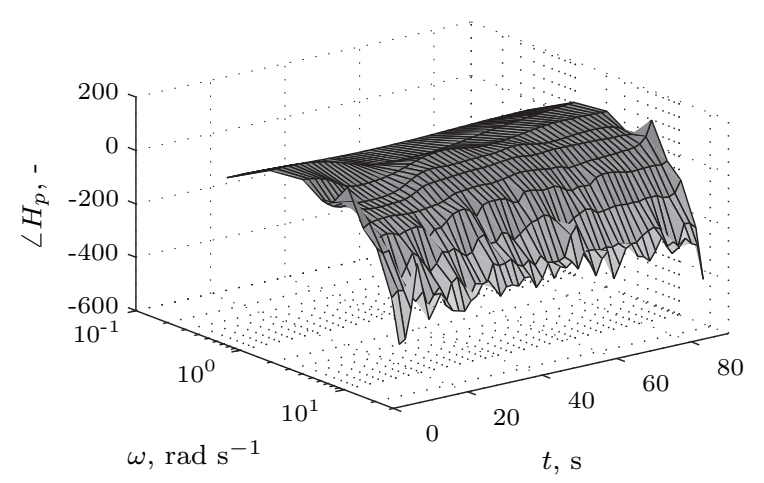

(b) MLE pilot magnitude

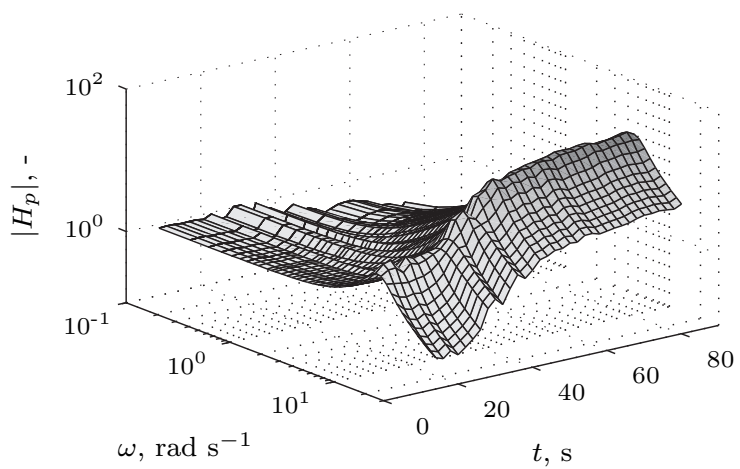

(d) MLE pilot phase

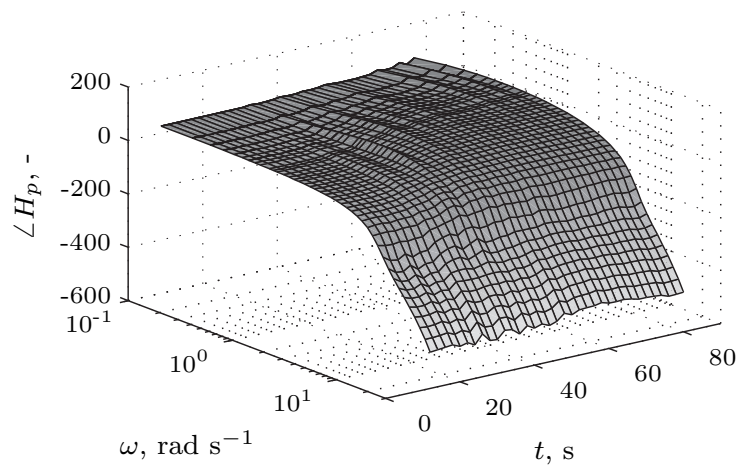

Figure 11: Time-varying pilot identification results from the wavelet and MLE methods $\left(P_{n}=0.05\right)$.

closely follow the simulated values. The edge effects of the wavelet estimates in Figure 9 also slightly affect the wavelet estimates of the crossover frequency and phase margin at the edges. The crossover frequencies and phase margins resulting from the MLE data show a large discrepancy around $t=30.0 \mathrm{~s}$. This coincides with the error in the estimated frequency responses observed in Figures 9 and 10. At $t=70.0 \mathrm{~s}$ where the change in dynamics is more gradual, the values are estimated correctly.

The estimated crossover frequencies and phase margins from the simulation data with pilot remnant are given in Figure 13. This figure again shows that the data resulting from the wavelet estimates is mostly affected by the addition of pilot remnant. The error in the MLE frequency response estimates at $t=30.0 \mathrm{~s}$ is still present.

\section{Parameter Estimates}

The parameters of the pilot model structure defined in Eq. (16) were estimated using the wavelet pilot frequency response estimates and the cost function defined in Eq. (9). The parameters were estimated at a two second interval to reduce the computational effort. Note that this is a second step to be performed after the calculation of the frequency responses using wavelets. The MLE procedure directly estimates the pilot model parameters from the time-domain data. For both parameter estimation techniques, the initial parameter vector at every time instance was chosen to be the simulated set of parameters. The wavelet and MLE parameter estimation results for all five parameters of the pilot model are given in Figure 14. These results are for a simulation without simulated pilot remnant.

From Figure 14 it can be observed that both techniques produce parameter estimation results that follow the general trends of the simulated parameters. The pilot gain resulting from the wavelet estimation procedure is estimated too low for almost the entire run. The MLE parameter estimates generally fluctuate around the simulated values. At the end of the simulation run, around $t=80.0 \mathrm{~s}$, the MLE estimates of pilot gain and lead time constant start to deviate from the real value.

The parameter estimation results for a run with simulated remnant is given in Figure 15. The remnant 
(a) crossover frequency

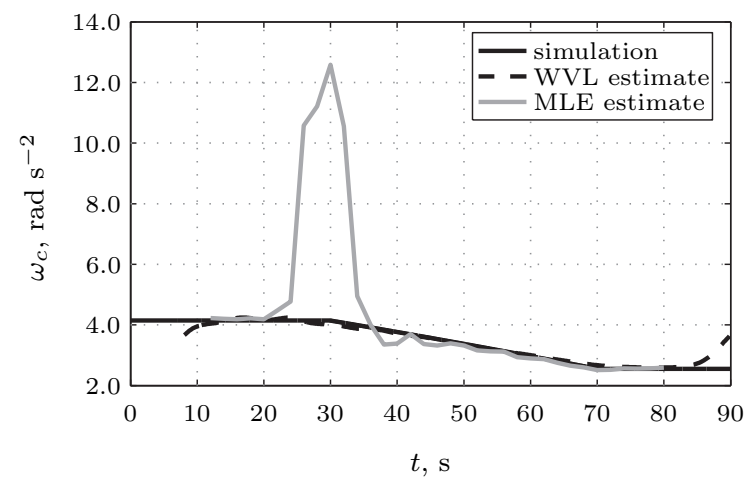

(b) phase margin

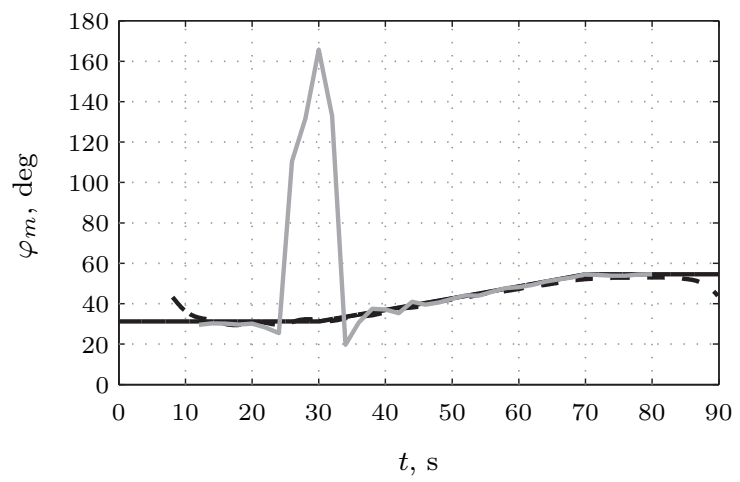

Figure 12: Estimated time-varying open-loop characteristics from wavelet and MLE methods $\left(P_{n}=0\right)$.

(a) crossover frequency

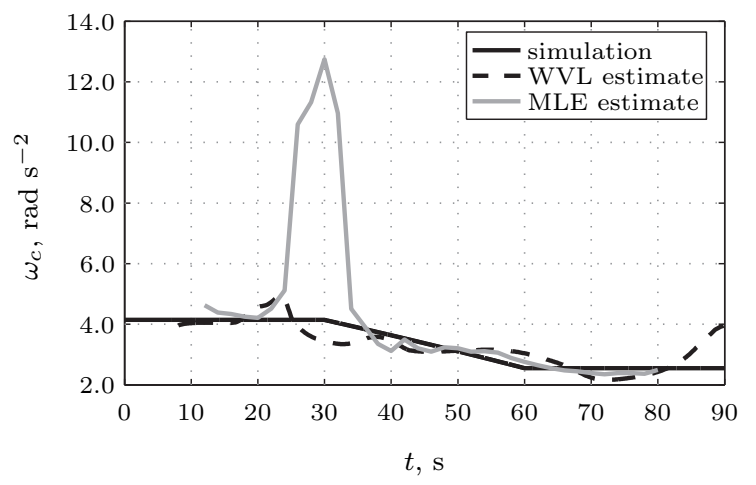

(b) phase margin

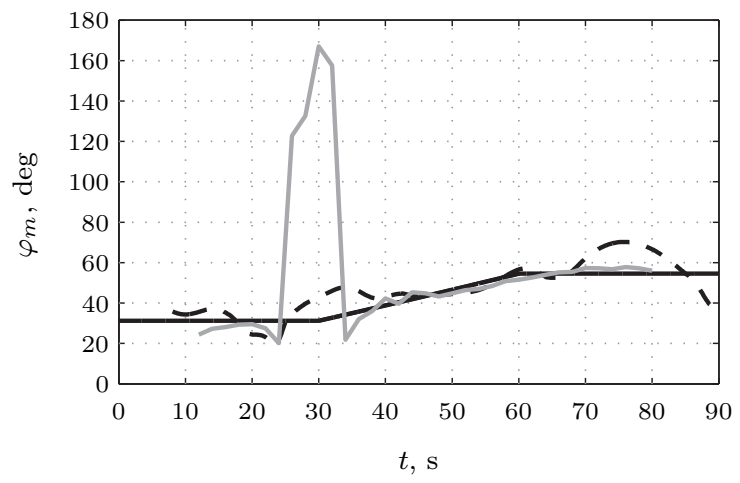

Figure 13: Estimated time-varying open-loop characteristics from wavelet and MLE methods $\left(P_{n}=0.05\right)$.

variance percentage was kept constant at $5 \%$ for the length of the simulation run. It can be observed that the estimates from both the wavelet and MLE procedure are affected by the remnant. The wavelet parameter estimates are mostly affected by the pilot remnant, especially before the start of the parameter transition $(t<30 \mathrm{~s})$. The lead time constant estimated using the two-step wavelet procedure starts to deviate from the middle of the run. The pilot gain and lead time constant resulting from the MLE procedure start to deviate from the end of the transition phase.

Simulation runs were performed with percentages of pilot remnant variance up to $20 \%$. However, no parameter estimates could be obtained using the two-step wavelet parameter estimation procedure. The bias and variance of the pilot frequency responses calculated using the wavelet transform proved to be too high to produce reliable parameter estimates in the second step. In most cases the constrained optimization procedure returned parameter values at the boundaries of the parameter search space. The MLE procedure was able to produce reliable parameter estimation results comparable to the results given in Figure 15.

\section{Discussion}

Simulations of a closed-loop manual control task with time-varying dynamics were performed to evaluate a two-step parameter estimation technique using wavelets and a one-step parameter estimation technique using MLE for the estimation of the time-varying pilot model parameters. The simulation was set up using knowledge of pilot performance and equalization characteristics. However, no parameter values from real experiments were taken. The use of simulations allowed for an objective comparison of both parameter estimation procedures and their sensitivity to pilot remnant. Future studies should investigate the use of both techniques in a human-in-the-loop experiment. 
(a) pilot gain

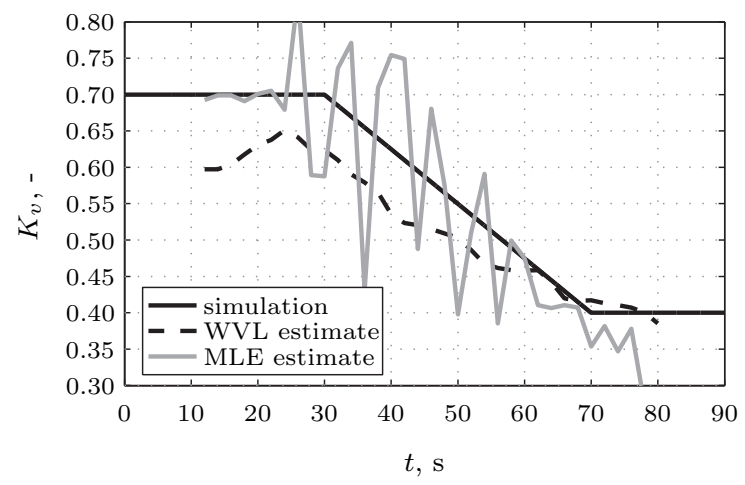

(c) time delay

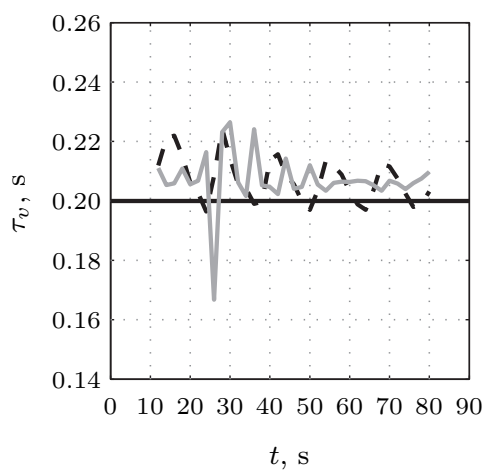

(d) neuromuscular damping

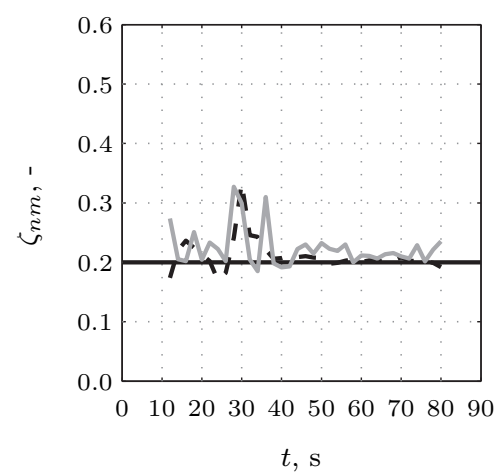

(b) lead time constant

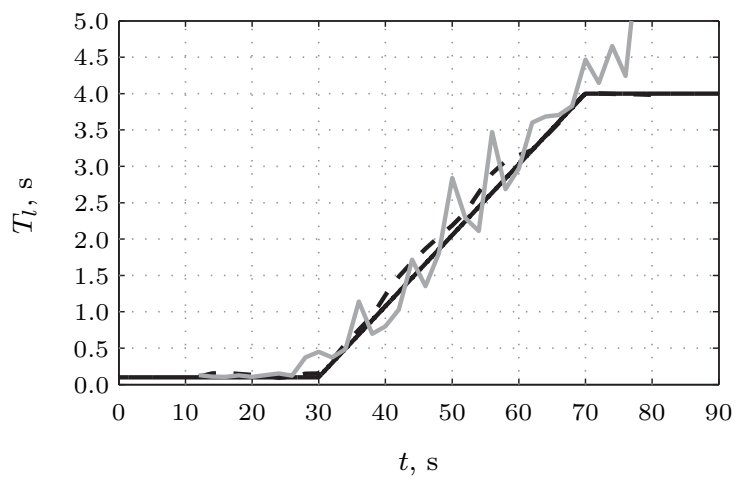

(e) neuromuscular frequency

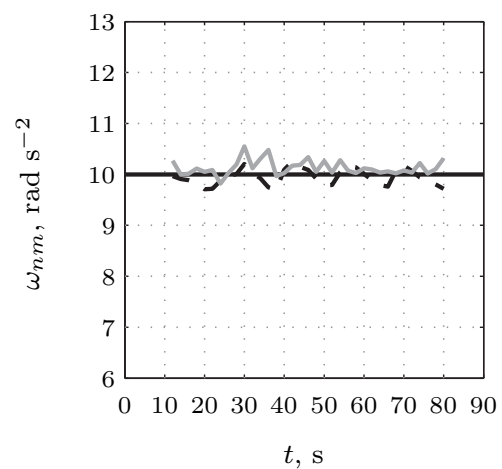

Figure 14: Estimated time-varying pilot model parameters from wavelet and MLE methods $\left(P_{n}=0\right)$.

The pilot remnant was simulated as a low-pass filtered Gaussian white noise signal. The time constant of the filter was kept constant. The variance percentage of the remnant in the pilot control signal was also constant during a simulation run. These values were kept constant to reduce the amount of variables changing at the same time to allow for a better evaluation of performance in estimating the time-varying pilot equalization parameters. However, in a real-life task, the characteristics of pilot remnant would change over time in correspondence to the changing vehicle dynamics. For example, the percentage of pilot remnant is generally higher when controlling double integrator dynamics (acceleration control) compared to single integrator dynamics (velocity control).

In the case of no simulated pilot remnant, the wavelet parameter estimation technique was able to provide accurate estimates of the time-varying pilot frequency response, and crossover frequencies and phase margins. The results were only slightly affected by the edge effects that are commonly seen when using wavelets. ${ }^{8}$ However, the frequency response functions identified using wavelets were greatly affected with the addition of simulated pilot remnant, even with a variance as low as $5 \%$ of the total pilot control signal variance. The parameter estimates resulting from the two-step wavelet parameter estimation technique proved to be unreliable in this case. With higher variances of pilot remnant, no parameter estimates could be obtained.

The performance of the wavelet parameter estimation method is dependent on the type of wavelet function and the selected wavelet parameters. The Morlet wavelet used in this study is the most commonly used wavelet function for the identification of human control behavior. ${ }^{9,10}$ However, depending on the task, other types of wavelet functions may provide better results. In addition, time averaging the wavelet transforms before the calculation of the pilot frequency response functions might improve the method's accuracy when applied to data with considerable pilot remnant. ${ }^{8}$ The averaging of the wavelet transform was not performed in the current study.

Without the addition of pilot remnant to the simulated control task, the MLE procedure provided accurate results for the pilot frequency responses and model parameters. However, the method proved to be less effective when the dynamics of the pilot are changing rapidly, as seen by the significant error between simulated and estimated response functions at $t=30.0 \mathrm{~s}$ in Figure 10. This is caused by the averaging effect 
(a) pilot gain

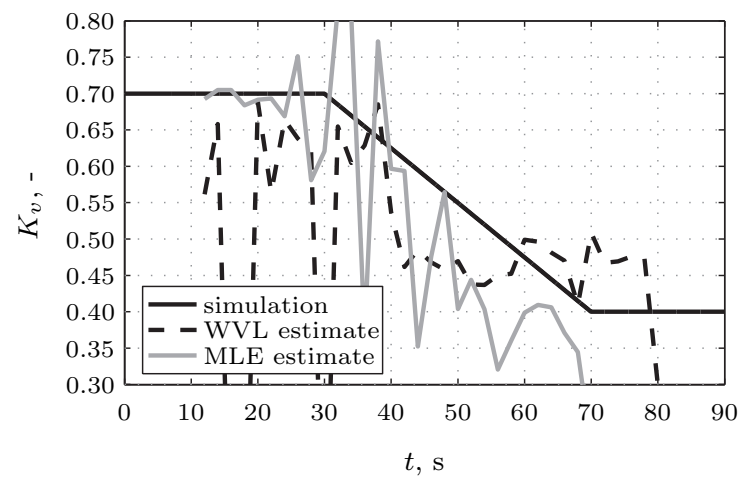

(c) time delay

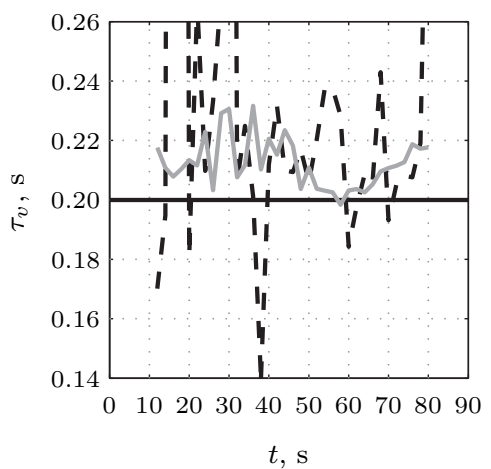

(d) neuromuscular damping

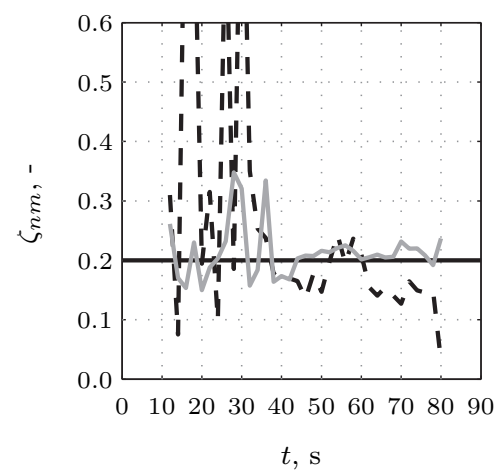

(b) lead time constant

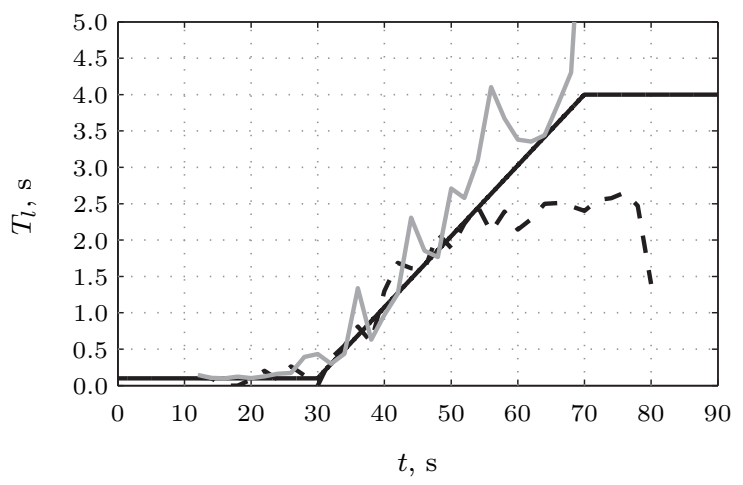

(e) neuromuscular frequency

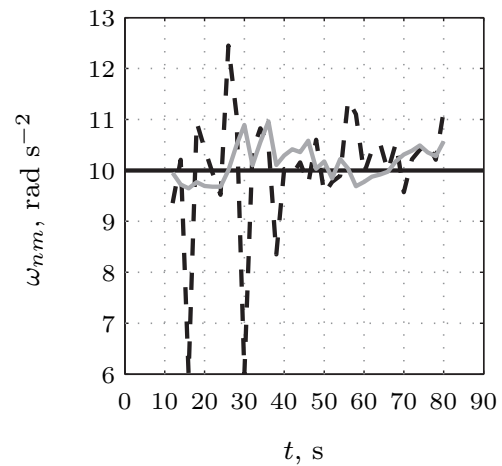

Figure 15: Estimated time-varying pilot model parameters from wavelet and MLE methods $\left(P_{n}=0.05\right)$.

of the moving time window. In general, the pilot frequency response and model parameter estimates from the MLE procedure were less affected by pilot remnant. This warrants the use of the MLE procedure for future studies concerning the estimation of time-varying pilot model parameters. However, more knowledge needs to be gained on the effects of the moving time-window length on the estimation results.

In the current study, only an unconstrained gradient-based Gauss-Newton optimization was performed to find the parameter estimate with the highest likelihood using MLE. In Reference 4, it was found that a genetic algorithm - in addition to the Gauss-Newton optimization - significantly improved the MLE method's capability of finding a global optimum solution. However, the genetic algorithm significantly increases the computational effort needed, especially when performed every time step. A solution could be to use the genetic algorithm to calculate an initial parameter vector at the start of a run only and use the results of the Gauss-Newton optimization as the initial parameter vector for the Gauss-Newton optimization in the next step. However, if the optimization at a single time instance would produce a non-optimal solution, it might not be possible to achieve global optimum solutions at future time steps.

To improve the performance of MLE for the identification of time-varying parameters in future studies, the method should be enhanced to estimate the parameters as a function of time directly as opposed to using a sliding time window. This would reduce the computational effort and increase accuracy. However, assumptions need to be made on the parameter functions and properties before the analysis. These assumptions can easily be made in an experiment where the controlled time-varying dynamics are known, but this will be more difficult in real-life applications.

Using both estimation techniques, the accuracy of the estimation results are highly dependent on the values of the pilot model parameters in combination with the frequency content of the forcing function. For example, when the inverse of the lead time constant of the pilot model decreases to a value below the lowest input frequency of the forcing function, the pilot model gain can be estimated less accurately. This problem can be minimized by careful design of the forcing functions and prior knowledge of the change in time-varying dynamics.

The current study was a first attempt to evaluate wavelets and MLE for the estimation of time-varying 
pilot model parameters. More research into the performance of both methods is needed. In addition, the methods need to be extended to a multi-loop case; that is, pilot models with different perceptual inputs. In addition, the variation of parameters other than the pilot equalization parameters, such as the pilot time delay and neuromuscular parameters, should be considered in future studies. Finally, the variation in parameters was assumed to be linear in the current study. Nonlinear variations in parameters should be considered in future research.

The estimation of a time-varying component of pilot control behavior parameters adds a very complex problem to the already difficult problem of pilot control behavior identification. Future experiments on time-varying pilot control behavior should be designed carefully. The accuracy of the methods discussed here should be evaluated more thoroughly before using the methods in experiments or real-life task analysis.

\section{Conclusions}

This paper presents two methods for the estimation of time-varying pilot model parameters, a twostep method using wavelets and a windowed maximum likelihood estimation method. The methods were evaluated using simulations of a closed-loop control task with time-varying pilot equalization and vehicle dynamics. Simulations were performed with and without remnant. Both methods give accurate results when no pilot remnant is present. The wavelet transform is very sensitive to measurement noise, resulting in inaccurate parameter estimates when considerable remnant is present. The MLE method is less sensitive to pilot remnant, but is unable to detect fast changes in pilot control behavior.

\section{References}

${ }^{1}$ McRuer, D. T., Graham, D., Krendel, E. S., and Reisener, W., "Human Pilot Dynamics in Compensatory Systems. Theory, Models and Experiments With Controlled Element and Forcing Function Variations," Tech. Rep. AFFDL-TR-65-15, Wright Patterson AFB (OH): Air Force Flight Dynamics Laboratory, 1965.

${ }^{2}$ Stapleford, R. L., Peters, R. A., and Alex, F. R., "Experiments and a Model for Pilot Dynamics with Visual and Motion Inputs," NASA Contractor Report NASA CR-1325, NASA, 1969.

${ }^{3}$ Nieuwenhuizen, F. M., Zaal, P. M. T., Mulder, M., van Paassen, M. M., and Mulder, J. A., "Modeling Human Multichannel Perception and Control Using Linear Time-Invariant Models," Journal of Guidance, Control, and Dynamics, Vol. 31, No. 4, July-Aug. 2008, pp. 999-1013.

${ }^{4}$ Zaal, P. M. T., Pool, D. M., Chu, Q. P., van Paassen, M. M., Mulder, M., and Mulder, J. A., "Modeling Human Multimodal Perception and Control Using Genetic Maximum Likelihood Estimation," Journal of Guidance, Control, and Dynamics, Vol. 32, No. 4, July-Aug. 2009, pp. 1089-1099.

${ }^{5}$ Hess, R. A., "Modeling Pilot Control Behavior with Sudden Changes in Vehicle Dynamics," Journal of Aircraft, Vol. 46, No. 5, Sept.-Oct. 2009, pp. 1584-1592.

${ }^{6}$ Young, L. R., "On Adaptive Manual Control," Ergonomics, Vol. 12, No. 4, 1969, pp. 635-675.

${ }^{7}$ Kumar, P. and Foufoula-Georgiou, E., "Wavelet Analysis for Geophysical Applications," Reviews of Geophysics, Vol. 35, No. 4, Nov. 1997, pp. 385-412.

${ }^{8}$ Torrence, C. and Compo, G. P., "A Practical Guide to Wavelet Analysis," Bulletin of the American Meteorological Society, Vol. 79, No. 1, Jan. 1998, pp. 61-78.

${ }^{9}$ Thompson, P. M., Klyde, D. H., and Brenner, M. J., "Wavelet-Based Time-Varying Human Operator Models," Proceedings of the AIAA Atmospheric Flight Mechanics Conference and Exhibit, Montreal (CA), No. AIAA-2001-4009, 6-9 Aug. 2001.

${ }^{10}$ Thompson, P. M., Klyde, D. H., Bachelder, E. N., Rosenthal, T. J., and Brenner, M. J., "Development of Wavelet-Based Techniques for Detecting Loss of Control," Proceedings of the AIAA Atmospheric Flight Mechanics Conference and Exhibit, Providence (RI), No. AIAA-2004-5064, Aug. 16-19 2004.

${ }^{11}$ Thompson, P. M. and Klyde, D. H., "Exploration of the Properties of Analytic Wavelets for Systems Analysis," Proceedings of the AIAA Atmospheric Flight Mechanics Conference and Exhibit, Monterey (CA), No. AIAA-2002-4707, Aug. 5-8 2002.

${ }^{12}$ Zaal, P. M. T. and Sweet, B. T., "Effects of Visual Spatio-Temporal Aliasing on Pilot Performance in Active Control Tasks," Proceedings of the AIAA Guidance, Navigation, and Control Conference and Exhibit, Toronto (ON), Canada, No. AIAA-2010-8096, 2-5Aug. 2010.

${ }^{13}$ Damveld, H. J., A Cybernetic Approach to Assess the Longitudinal Handling Qualities of Aeroelastic Aircraft, Doctoral dissertation, Faculty of Aerospace Engineering, Delft University of Technology, 2009.

${ }^{14}$ Hess, R. A., "Rudder Control Strategies and Force/Feel System Designs in Transport Aircraft," Journal of Guidance, Control, and Dynamics, Vol. 28, No. 6, Nov.-Dec. 2005, pp. 1251-1262.

${ }^{15}$ Zaal, P. M. T., Pool, D. M., Mulder, M., van Paassen, M. M., and Mulder, J. A., "Multimodal Pilot Model Identification in Real Flight," Proceedings of the AIAA Modeling and Simulation Technologies Conference and Exhibit, Chicago (IL), No. AIAA-2009-6028, 10-13Aug. 2009. 